\title{
Exosomal hsa-miR-933 in Gastric Juice as a Potential Biomarker for Functional Dyspepsia
}

Fumio Tanaka, Shingo Takashima, Yuji Nadatani, Koji Otani, Shuhei Hosomi, Noriko Kamata, Koichi Taira, Yasuaki Nagami, Tetsuya Tanigawa, Shinya Fukumoto, Toshio Watanabe, Yoshiki Murakami, Norifumi Kawada, Yasuhiro Fujiwara

\begin{tabular}{|c|c|}
\hline Citation & Digestive Diseases and Sciences. 65(12); 3493-3501 \\
\hline Issue Date & 2020-01-23 \\
\hline Type & Journal Article \\
\hline Textversion & Author \\
\hline Rights & $\begin{array}{l}\text { This is a post-peer-review, pre-copyedit version of an article published in Digestive } \\
\text { Diseases and Sciences. The final authenticated version is available online at: } \\
\text { https://doi.org/10.1007/s10620-020-06096-7. } \\
\text { See Springer Nature terms of use. } \\
\text { https://www.springer.com/gp/open-access/publication-policies/aam-terms-of-use }\end{array}$ \\
\hline DOI & $10.1007 / \mathrm{s} 10620-020-06096^{-7}$ \\
\hline
\end{tabular}

\author{
Self-Archiving by Author(s) \\ Placed on: Osaka City University
}

Tanaka, F., Takashima, S., Nadatani, Y. et al. (2020). Exosomal hsa-miR-933 in Gastric Juice as a Potential Biomarker for Functional Dyspepsia. Digestive Diseases and Sciences. DOI: 
Exosomal hsa-miR-933 in gastric juice as a potential biomarker for functional dyspepsia

Fumio Tanaka ${ }^{1,2}$, Shingo Takashima ${ }^{1}$, Yuji Nadatani ${ }^{1,2}$, Koji Otani $^{1,2}$, Shuhei Hosomi ${ }^{1}$, Noriko Kamata $^{1}$, Koichi Taira ${ }^{1}$, Yasuaki Nagami ${ }^{1}$, Tetsuya Tanigawa ${ }^{1}$, Shinya Fukumoto ${ }^{2}$, Toshio Watanabe $^{1}$, Yoshiki Murakami ${ }^{3}$, Norifumi Kawada ${ }^{2,4}$, and Yasuhiro Fujiwara ${ }^{1}$

${ }^{1}$ Department of Gastroenterology, Graduate School of Medicine, Osaka City University, Osaka, Japan

${ }^{2}$ Department of Premier Preventive Medicine, Graduate School of Medicine, Osaka City University, Osaka, Japan

${ }^{3}$ Department of Molecular Pathology, Tokyo Medical University, Tokyo, Japan

${ }^{4}$ Department of Hepatology, Graduate School of Medicine, Osaka City University, Osaka, Japan

Corresponding Author: Fumio Tanaka, MD, PhD

Department of Gastroenterology, Graduate School of Medicine, Osaka City University, 1-4-3 Asahimachi, Abeno-ku, Osaka 545-8585, Japan

Phone: +81-6-6645-3811; Fax: +81-6-6645-3813;

E-mail: m2079981@med.osaka-cu.ac.jp

Short running title: MicroRNAs in functional dyspepsia

Grant support: This work was supported by JSPS KAKENHI Grant Number JP16K09252.

Conflict of interest: The authors state that they are no competing interests to declare. 


\section{Abstract}

Background: MicroRNAs (miRNAs) in exosomes represent disease-specific profiles, and are applied as biomarkers in oncology. However, in functional dyspepsia (FD), the role of exosomal miRNAs has not been fully elucidated.

Aims: To investigate exosomal miRNAs as potential biomarkers of FD using liquid biopsy.

Methods: This retrospective cohort study included 11 subjects with FD and 11 age- and sexmatched healthy controls (HCs). We collected gastric juice and isolated exosomal miRNAs. In a discovery cohort, expression levels of 2,565 miRNAs were evaluated by 3D-Gene ${ }^{\circledR}$ microarray. miRNA expression profiles from exosomes of subjects with FD and HCs were compared by two normalization methods: (1) global normalization and (2) normalization by internal control. Subsequently, in a validation cohort, the expression levels of miRNAs were validated by quantitative reverse transcription PCR (RT-qPCR).

Results: Through microarray analysis using the two methods, we identified 39 miRNAs that were consistently and significantly downregulated in FD cases compared with those in HCs. Of these, 12 miRNAs (hsa-miR-933, -345-5p, -708-5p, -203a-3p, -619-5p, -4294, -4481, 196a-5p, -3918, -372-3p, -658, and -3654) were further validated by RT-qPCR. Our results indicated that hsa-miR-933 was significantly downregulated in FD compared with HCs $(0.317$ \pm 0.205 -fold, $P=0.0317$ ). Furthermore, the expression level of hsa-miR-933 was negatively associated with dyspepsia score and the frequency of epigastric pain and/or burning $(P<0.01$, $r=-0.835 ; P=0.0280, r=-0.688$, respectively).

Conclusions: Exosomal hsa-miR-933 in gastric juice could be a candidate biomarker for FD.

Keywords: Biomarkers, Circulating MicroRNA, Dyspepsia, Gastric juice, MicroRNAs 


\section{Introduction}

Functional dyspepsia (FD) is a multifactorial disorder associated with gastrointestinal (GI) motor dysfunction, visceral hypersensitivity, microinflammation, intestinal permeability, and psychosocial factors [1-4]. In terms of therapeutic options for FD, acid secretion inhibitors (such as proton-pump inhibitors (PPIs)), prokinetics (such as acotiamide), and tricyclic antidepressants are commonly used according to guidelines of western and eastern countries $[5,6]$. However, their therapeutic efficacies have not been fully satisfactory because the pathophysiology of FD is heterogeneous and complex. In other words, there is the possibility that heretofore unrecognized factors influence the development of FD. Accordingly, clarifying new pathophysiology has been warranted for advancing the understanding of FD. Moreover, only a few biomarkers of FD have been identified that can aid understanding of the status of patients. Identifying a biomarker using a minimally invasive method is also warranted.

Recently, liquid biopsy has emerged as an effective and minimally invasive method to diagnose and monitor diseases using biological samples, such as blood, urine, and saliva [7-9]. As a minimally invasive method to obtain samples from patients with FD, we focused on gastric juice. Gastric juice is easy to obtain through esophagogastroduodenoscopy (EGD) without damaging any tissues. Gastric juice contains exosomes that contain mediators, such as microRNAs (miRNAs), for intercellular communication [10]. Exosomes are released from various types of cells and circulate in body fluids to perform cell-to-cell communication [11]. miRNAs are small non-coding RNAs with sizes ranging from 19-25 nucleotides and they play important roles in inhibitory regulation of target genes [12]. They can bind to target messenger RNAs (mRNAs) in 3'-untranslated regions or to imperfect complementary sequences called miRNA response elements, which leads to mRNA degradation or translational inhibition [13]. As a characteristic of miRNAs in exosomes, they represent disease-specific profiles and are highly stable and reproducible in biological samples. For example, previous oncology studies 
have shown that exosomal miRNAs have potential as useful biomarkers for diagnosis, metastasis, and drug resistance [14-16]. Moreover, we previously reported that the expression level of exosomal miRNA is associated with the grade of inflammation and stage of liver fibrosis in chronic liver disease [17]. However, in FD, the role of exosomal miRNAs has not been fully elucidated. To date, there are limited studies that have attempted to use miRNA in the context of FD. In a rat model of FD, the expression level of miR-19a in gastric tissue was higher than that of normal rats, and reduced miR-19a could ameliorate GI motility [18]. In one human study, genetic polymorphism of pri-miR-325 was closely associated with the risk of FD in Japan [19]. Therefore, we aimed to investigate exosomal miRNAs as potential biomarkers of FD by liquid biopsy.

\section{Methods}

\section{Outline of this study}

This retrospective cohort study included 11 subjects with FD identified based on Rome III criteria and 11 age- and sex-matched healthy controls (HCs). All participants tested negative for Helicobacter pylori (HP) infection. We collected $10 \mathrm{~mL}$ of gastric juice by EGD and isolated exosomal miRNAs. We used the samples for the following experiments. First, as a discovery set, we randomly selected six subjects with FD and six HCs. Next, the expression levels of 2,565 miRNAs were evaluated by microarray to discover candidate biomarkers of FD. Expression profiles of subjects with FD and HCs were then compared by two methods: 1 . Global normalization 2. Correction by internal control, which had the smallest coefficient of variation $(\mathrm{CV})$. Second, as a validation set, we randomly selected five subjects with FD and five HCs. In this set, we validated candidate miRNAs found on the screen using quantitative reverse transcription polymerase chain reaction (RT-qPCR). 


\section{Participants}

Between July 2015 and February 2016, a total of 441 consecutive subjects underwent medical health check-ups, including EGD and blood examinations for anti-HP immunoglobulin G antibody, at our clinic, MedCity21. Among participants, we diagnosed subjects with FD and HCs. The inclusion criteria were as follows: above 18 years of age, and subjects who provided comprehensive written informed consent for biorepository and gene analysis. The exclusion criteria were as follows: severe comorbidities (GI, heart, lung, liver, kidney, endocrine, gynecological, and mental diseases), subjects who presented a history of any type of cancer, history of abdominal surgery, subjects taking drugs (PPI, histamine $\mathrm{H}_{2}$ receptor antagonists $\left(\mathrm{H}_{2} \mathrm{RA}\right)$, steroids, immunosuppressive drugs, nonsteroidal anti-inflammatory drugs (NSAIDs), and anti-coagulants), and detection of organic diseases on EGD (ulcer, acute gastric mucosal lesions, and neoplastic lesions). We also excluded subjects with current or previous HP infection, including subjects with false-negative anti-HP antibody results.

The study protocol was in accordance with the Declaration of Helsinki and was approved by the ethics committee of the Osaka City University Graduate School of Medicine (protocol number 3438). All participants gave written informed consent. We disclosed the information of this study on our home page and provided the opportunity to opt out of the study. This study was registered with the university hospital medical information network (UMIN) clinical trial registry (UMIN000022824). The following information was obtained from our medical records: age, gender, body mass index (BMI), current alcohol drinking, and current cigarette smoking status.

\section{Diagnosis of FD and HCs}

FD was diagnosed using Rome III criteria. We assessed four dyspepsia symptoms according to the Rome III criteria: postprandial fullness, early satiation, epigastric pain, and epigastric 
burning. ${ }^{1}$ We used our original questionnaire to assess the frequency of the above-mentioned dyspepsia symptoms according to a well-validated questionnaire in Japanese [20]. Symptoms were evaluated by a seven-point Likert scale: 0 , none; $1,<1$ day per month; 2,1 day per month; 3, 2-3 days per month; 4, 1 day per week; 5, > 1 day per week; and 6 , every day. We also evaluated dyspepsia and gastroesophageal reflux symptoms using a modified frequency scale for the symptoms of gastroesophageal reflux disease (FSSG), which is also a well-validated questionnaire in Japanese [21]. This questionnaire includes an evaluation of dyspepsia score (range, 0-28) and gastroesophageal reflux score (range, 0-28). We considered subjects who did not meet Rome III criteria for FD as HCs.

\section{Diagnosis of HP status}

We recorded history of past HP infection and eradication. We also used anti-HP antibody for the serological diagnosis of HP infections (E plate Eiken HP antibody ${ }^{\circledR}$; Eiken Chemical Co., Ltd., Tochigi, Japan). The cutoff value for the anti-HP antibody was set at $10 \mathrm{U} / \mathrm{mL}$. We considered the results as false-negative for anti-HP antibody when subjects demonstrated endoscopic findings compatible with those of current HP infections and with antibody values

$<10 \mathrm{U} / \mathrm{mL}$. Endoscopic findings of HP infection were diagnosed according to the Kyoto classification of gastritis [22]. The findings of current HP infections were as follows: diffuse redness, enlarged fold, nodularity, mucosal swelling, sticky mucus, and gastric atrophy.

\section{Isolation of exosomes from gastric juice}

Samples of $10 \mathrm{~mL}$ of gastric juice were stored at $-20^{\circ} \mathrm{C}$ until RNA preparation. Samples were centrifuged at $3000 \times g$ for $15 \mathrm{~min}$ at $4^{\circ} \mathrm{C}$ to remove cells and cellular fragments. Supernatants were mixed with $2 \mathrm{~mL}$ of ExoQuick-TC (System Biosciences, CA, USA), and exosomes were precipitated overnight at $4^{\circ} \mathrm{C}$. Exosome pellets were collected by centrifugation at $3000 \times g$ for 
$30 \mathrm{~min}$ at $4^{\circ} \mathrm{C}$ and then dissolved in $300 \mu \mathrm{L}$ of phosphate-buffered saline.

\section{miRNA microarray}

We performed miRNA microarray assays using 3D-Gene ${ }^{\circledR}$ (Toray Industries, Inc. Tokyo, Japan) on a discovery set. We compared the expression levels of 2,565 miRNAs between FD and HCs to detect candidate biomarkers of FD. To obtain robustness, we analyzed expression profiles by using two methods. In method 1, we performed global normalization. In global normalization, the correction that adjusts the median value of signal intensity was 25 . In method 2, correction by internal control was performed. The miRNA with the lowest CV in the microarray analysis was used as an internal control. miRNA signal values were standardized to the ratio of the average signal of the internal control. Finally, we confirmed the miRNAs that were consistently altered in subjects with FD compared with those in HCs by the abovementioned two means. This microarray assay was performed using the same protocol indicated in previous reports $[23,24]$. Briefly, total RNA was extracted from exosomes using the 3DGene ${ }^{\circledR}$ RNA extraction reagent, and fluorescent labeling was performed. RNA was hybridized to the $3 \mathrm{D}-\mathrm{Gene}{ }^{\circledR}$ Human miRNA Oligo Chip designed to detect 2,565 miRNA sequences registered in miRBase release 21 (http://www.mirbase.org/). This chip was scanned using a 3D-Gene ${ }^{\circledR}$ Scanner. The microarray data were obtained in accordance with the Minimum Information About a Microarray Experiment (MIAME) guidelines [25].

\section{Validation of microarray by RT-qPCR}

On a validation set, we performed RT-qPCR to validate the expressions of candidate exosomal miRNAs identified by microarray as biomarkers of FD. We selected candidate miRNAs with a $P$ value $<0.01$ on microarray. Furthermore, we also selected candidate miRNAs which had known target genes related to epithelial or neural function based on the databases of miRNAs, 
such as miRDB (http://mirdb.org) and miRTarBase (http://mirtarbase.mbc.nctu.edu.tw/). Next, we performed Taqman advanced miRNA assays. Total RNA was extracted from exosomes using MiRNeasy mini kit according to the manufacturer's instruction (Qiagen, Tokyo, Japan). cDNA was generated using Taqman miRNA reverse transcription kit. RT-qPCR was performed using Applied Biosystems 7500 Fast Real-Time PCR system with Taqman Fast universal PCR master mix. All PCR primers and Taqman probes for each miRNA were purchased from Thermo Fisher Scientific K.K. (Tokyo, Japan). The thermal cycling protocol was 50 cycles of amplification at $95^{\circ} \mathrm{C}$ for $15 \mathrm{~s}$ and $60^{\circ} \mathrm{C}$ for $1 \mathrm{~min}$. The miRNA with the lowest $\mathrm{CV}$ in the microarray analysis was used as an internal control. Relative levels of mRNA expression were calculated by indicating the mean value of HCs as 1 .

\section{Statistical analysis}

Data are expressed as median and interquartile range for continuous variables, and as numbers for categorical variables for clinical characteristics. For categorical variables, comparisons were performed using Fisher's exact test, whereas continuous variables were compared by Mann-Whitney U test. For RT-qPCR, data are expressed as mean \pm standard deviation. $P$ values $<0.05$ were considered statistically significant. Spearman's rank correlation coefficients were calculated for correlation analyses. All statistical analyses were performed using EZR (version 1.34; Saitama Medical Center, Jichi Medical University, Saitama, Japan), a graphical user interface for R (version 3.3.2; The R Foundation for Statistical Computing, Vienna, Austria) [26].

\section{Results}

\section{Detection of FD and HCs}

A flow chart of the study is shown in Fig. 1. Of the 441 subjects, 95 were excluded owing to 
the following criteria: severe comorbidity $(n=7)$, history of abdominal surgery $(n=59)$, current medication ( $\mathrm{n}=25 ; 10$ on PPI, 3 on $\mathrm{H}_{2} \mathrm{RA}, 1$ on steroids, 5 on NSAIDs, and 6 on anticoagulants), and gastric cancer detected on EGD $(n=4)$. Furthermore, we also excluded subjects with current or previous HP infection $(n=114)$. Of the remaining 232 subjects, we diagnosed 11 subjects with FD. Of 221 subjects categorized as HCs, we randomly selected 11 age- and sex-matched subjects. Finally, 11 subjects with FD and 11 HCs were analyzed in the study.

\section{Clinical characteristics of the study population by microarray analysis}

Clinical characteristics of the study population in the microarray analysis are shown in Table 1. A significantly higher frequency of postprandial fullness and epigastric pain/burning was observed in subjects with FD than in $\operatorname{HCs}(3.5[3.0,4.8]$ vs. $0.0[0.0,0.0], P<0.01 ; 4.0[4.0$, 4.8] vs. 0.0 [0.0, 0.0$], P<0.01$, respectively). Moreover, subjects with FD had significantly higher total and dyspepsia scores on modified FSSG than those of HCs $(10.5[9.3,13.3]$ vs. 0.5 $[0.0,1.0], P<0.01 ; 7.5[6.3,8.8]$ vs. $0.5[0.0,1.0], P<0.01$, respectively). These results confirmed that subjects with FD had more severe symptoms than those of HCs. However, there were no differences in the frequency of early satiation $(0.0[0.0,0.0]$ vs. $0.0[0.0,0.0], P=$ 0.405). There were no significant differences between subjects with FD and HCs with respect to other factors, such as age, gender, BMI, current cigarette smoking status, and current alcohol drinking.

\section{Microarray analysis}

The results of microarray analysis are shown in Fig. 2 and Table 2. In method 1, microarray analysis using global normalization identified 57 mRNAs that were significantly downregulated in FD compared with those in HCs. However, one miRNA was significantly 
upregulated in FD. Because the miRNA with the lowest CV was hsa-miR-6759-5p, we used hsa-miR-6759-5p as an internal control in method 2. Microarray analysis with correction by hsa-miR-6759-5p identified 43 significantly downregulated miRNAs in FD. Two miRNAs were significantly upregulated in FD. Therefore, we observed that 39 miRNAs were consistently downregulated in FD in the two experiments (Table 2). Of these, 12 miRNAs (hsamiR-619-5p, -3918, -372-3p, -4294, -708-5p, -3654, -4481, -203a-3p, -196a-5p, -933, -345-5p, and -658) were further validated by RT-qPCR. We selected 7 candidate miRNAs with $P$ value $<0.01$ on microarray assays (hsa-miR-619-5p, -3918, -372-3p, -4294, -708-5p, -3654, and 4481). We also selected 5 miRNAs which had known target genes related to epithelial or neural function (hsa-miR-203a-3p, -196a-5p, -933, -345-5p, and -658). Moreover, one miRNA (hsamiR-4745-3p) was consistently upregulated in FD (Table 2). hsa-miR-4745-3p was also further validated by RT-qPCR.

\section{Clinical characteristics of the study population by RT-qPCR analysis}

We next performed RT-qPCR on the validation set. The clinical characteristics of the set are shown in Table 3. Similar to that observed in the discovery set, a significantly higher frequency of postprandial fullness and epigastric pain/burning was observed in subjects with FD than in HCs $(4.0[4.0,5.0]$ vs. $0.0[0.0,1.0], P<0.01 ; 4.0[4.0,5.0]$ vs. $0.0[0.0,0.0], P<0.01$, respectively). The total and dyspepsia scores on modified FSSG of FD were also significantly higher than those of HCs $(17.0[17.0,23.0]$ vs. $2.0[0.0,3.0], P=0.0117 ; 11.0[8.0,13.0]$ vs. $1.0[0.0,1.0], P=0.0117$, respectively). Age, BMI, gender, current cigarette smoking status, current alcohol drinking, and frequency of early satiation were not different between FD and HCs.

\section{Validation of microarray by RT-qPCR}


The results of RT-qPCR are shown in Table 4. By RT-qPCR, hsa-miR-933 was significantly downregulated in FD compared with that in HCs $(0.317 \pm 0.205$ vs. $1.000 \pm 0.522$-fold, $P=$ 0.0317). As the downregulation of hsa-miR-933 as determined by microarray was wellvalidated by RT-qPCR, these results indicated that exosomal hsa-miR-933 could be a candidate biomarker for FD. Subjects with FD trended toward downregulation of hsa-miR-619-5p on RTqPCR analysis compared with HCs; however, this was not statistically significant $(0.604 \pm$ 0.295 vs. $1.000 \pm 0.303$-fold, $P=0.0556$ ). The expression of other miRNAs, including hsamiR-345-5p, -708-5p, -203a-3p, -4294, -4745-3p, -4481, -196a-5p, -3918, -372-3p, -658, and -3654, were also not statistically different between FD and HCs.

\section{Correlation analysis between hsa-miR-933 and clinical symptoms}

By correlation analysis, the expression level of hsa-miR-933 was negatively correlated with total and dyspepsia scores on modified FSSG $(P<0.01, r=-0.774$, Fig. 3a; $P<0.01, r=$ -0.835 , Fig. 3b, respectively). Furthermore, the expression level of hsa-miR-933 was also negatively correlated with the frequency of epigastric pain/burning $(P=0.0280, r=-0.688$, Fig. 3c). The level of hsa-miR-933 trended toward a negative correlation with the frequency of postprandial fullness $(P=0.0511, r=-0.630$, Fig. 3d $)$.

\section{Discussion}

In this study, we found that the expression of exosomal hsa-miR-933 in gastric juice was significantly downregulated in subjects with FD compared with that in HCs, both in the discovery and validation sets. Moreover, the expression level of hsa-miR-933 was negatively correlated with dyspepsia symptoms, such as epigastric pain and/or burning. These results indicated that hsa-miR-933 could be a novel candidate biomarker for FD. To our knowledge, this is the first report that identified candidate biomarkers using exosomal miRNAs in gastric 
juice. A previous study showed that miR-19a is associated with GI motility in a rat model of FD [18]. Arisawa et al. reported that genetic polymorphism of pri-miR-325 is associated with the risk of FD [19]. From our results, we identified hsa-miR-933 as a novel miRNA correlated with the pathophysiology of FD in humans, which was not found in previous reports.

To date, the biological functions of hsa-miR-933 remain unclear. To explore the roles of hsamiR-933 in the pathophysiology of FD, we referred to a miRNA database, miRDB, a renowned database for miRNA target prediction and functional annotation $[27,28]$. We found that, there were 33 predicted targets for hsa-miR-933 in miRDB. These results indicated that there was a possibility that hsa-miR-933 has multiple biological functions in humans. To research one of the biological roles of hsa-miR-933, we focused on the predicted target rank number 1. miRDB showed that predicted target number 1 for hsa-miR-933 was brain-derived neurotrophic factor (BDNF).

BDNF has a variety of potential ways to contribute to FD. BDNF is the most abundant neurotrophic factor in the brain [29]. Moreover, BDNF plays certain roles in neuronal survival, maintenance of neurotransmission, and in striatal serotonin fiber sprouting [30, 31]. Therefore, BDNF is considered to be involved in the pathophysiology of mood disorders, such as depression [32]. In the GI tract, BDNF acts as a neuromodulator of GI motor and sensory functions $[33,34]$. The administration of recombinant BDNF accelerates colonic transit in healthy individuals. BDNF contributes to colonic hypersensitivity via activating the enteroglianerve unit. Furthermore, Yu et al. reported that BDNF modulates the intestinal barrier function by regulating tight junction proteins [35]. In summary, BDNF may play a crucial role in the pathophysiology of FD, including the modulation of hsa-miR-933.

In this study, we observed decreased exosomal hsa-miR-933 expression in gastric juice. The source of exosomes containing hsa-miR-933 may be cells localized in the mucosal layer because exosomes were released into the gastric lumen and were present in gastric juice; gastric 
epithelial cells, neuron terminal innervated to epithelial cells, enteric glial cells, and inflammatory cells may be candidate cellular sources. Moreover, we found that the level of hsa-miR-933 was negatively correlated with symptoms of dyspepsia. In other words, hsa-miR933 may play roles in gastric hypersensitivity and barrier integrity. Decreased exosomal hsamiR-933 expression may nullify the inhibitory effects of BDNF.

There are certain limitations to this study. Although we performed a validation study, the sample size was very small. It would be appropriate and helpful to confirm the findings of this investigation in a larger study population. In a larger study, there is a possibility that hsa-miR619-5p may also function as a biomarker of FD because this miRNA was slightly beyond statistical significance in our results. A combination of candidate miRNAs may contribute to improved accuracy. Moreover, this study was conducted in a single center; therefore, a multicenter study is also warranted. Although collection of gastric juice is minimally invasive without injuring any tissues, we need to perform EGD, which is an invasive method. Therefore, in the future, we need to investigate additional non-invasive methods, such as collection of saliva.

In conclusion, exosomal hsa-miR-933 in gastric juice could be a candidate biomarker for FD. A future, prospective, larger study is warranted to confirm these results.

\section{Acknowledgements}

This work was supported by JSPS KAKENHI Grant Number JP16K09252. We thank the experimental support staff (Naomi Sakama, Emi Yoshioka, and Saori Itami) and nursing staff at MedCity21.

\section{Compliance with ethical standards}

\section{Disclosure of potential conflicts of interest}


The authors state that they have no conflicts of interest to declare.

\section{Research involving human participants}

Ethical approval: All procedures performed in studies involving human participants were in accordance with the ethical standards of the institutional research committee, and with the 1964 Helsinki declaration and its later amendments or comparable ethical standards. The study protocol was approved by the ethics committee of the Osaka City University Graduate School of Medicine (protocol number 3438).

\section{Informed consent}

All participants gave written informed consent. We disclosed information regarding this study on our home page and provided participants the opportunity to opt out of the study. 


\section{References}

1. Stanghellini V, Chan FK, Hasler WL, et al. Gastroduodenal Disorders. Gastroenterology. $2016 ; 150: 1380-1392$.

2. Miwa H, Oshima $\mathrm{T}$, Tomita $\mathrm{T}$, et al. Recent understanding of the pathophysiology of functional dyspepsia: role of the duodenum as the pathogenic center. $J$ Gastroenterol. 2019;54:305-311.

3. Tanaka F, Tominaga K, Fujikawa Y, et al. Concentration of Glial Cell Line-Derived Neurotrophic Factor Positively Correlates with Symptoms in Functional Dyspepsia. Dig Dis Sci. 2016;61:3478-3485.

4. Tominaga K, Higuchi K, Iketani $\mathrm{T}$, et al. Comparison of gastrointestinal symptoms and psychological factors of functional dyspepsia to peptic ulcer or panic disorder patients. Inflammopharmacology. 2007;15:84-89.

5. Moayyedi P, Lacy BE, Andrews CN, et al. ACG and CAG Clinical Guideline: Management of Dyspepsia. Am J Gastroenterol. 2017;112:988-1013.

6. Miwa H, Kusano M, Arisawa T, et al. Evidence-based clinical practice guidelines for functional dyspepsia. J Gastroenterol. 2015;50:125-139.

7. Murakami Y, Toyoda H, Tanahashi $\mathrm{T}$, et al. Comprehensive miRNA expression analysis in peripheral blood can diagnose liver disease. PLoS One. 2012;7:e48366.

8. Kutwin P, Konecki T, Borkowska EM, Traczyk-Borszyńska M, Jabłonowski Z. Urine miRNA as a potential biomarker for bladder cancer detection - a meta-analysis. Cent European J Urol. 2018;71:177-185.

9. Arantes LMRB, De Carvalho AC, Melendez ME, Lopes Carvalho A. Serum, plasma and saliva biomarkers for head and neck cancer. Expert Rev Mol Diagn. 2018;18:85-112.

10. Kagota S, Taniguchi K, Lee SW, et al. Analysis of Extracellular Vesicles in Gastric Juice from Gastric Cancer Patients. Int J Mol Sci. 2019;20. pii: E953. 
11. Lin J, Li J, Huang B, et al. Exosomes: novel biomarkers for clinical diagnosis. ScientificWorldJournal. 2015;2015:657086.

12. Nedaeinia R, Manian M, Jazayeri $\mathrm{MH}$, et al. Circulating exosomes and exosomal microRNAs as biomarkers in gastrointestinal cancer. Cancer Gene Ther. 2017;24:48-56.

13. Gregory RI, Yan KP, Amuthan G, et al. The Microprocessor complex mediates the genesis of microRNAs. Nature. 2004;432:235-240.

14. Huang Z, Huang D, Ni S, et al. Plasma microRNAs are promising novel biomarkers for early detection of colorectal cancer. Int J Cancer. 2010;127:118-126.

15. Zhow W, Fong MY, Min Y, et al. Cancer-secreted miR-105 destroys vascular endothelial barriers to promote metastasis. Cancer Cell. 2014;25:501-515.

16. Chen WX, Liu XM, Lv MM, et al. Exosomes from drug-resistant breast cancer cells transmit chemoresistance by a horizontal transfer of microRNAs. PLoS One. 2014;9:e95240.

17. Murakami Y, Toyoda H, Tanahashi T, et al. Comprehensive miRNA expression analysis in peripheral blood can diagnose liver disease. PLoS One. 2012;7:e48366.

18. Deng Y, Zhou X, Xiang X, Ou Y, He J. Effect of miRNA-19a on gastrointestinal motility in rats with functional dyspepsia. Exp Ther Med. 2018;15:4875-4879.

19. Arisawa T, Tahara T, Fukuyama T, et al. Genetic polymorphism of pri-microRNA 325, targeting SLC6A4 3'-UTR, is closely associated with the risk of functional dyspepsia in Japan. J Gastroenterol. 2012;47:1091-1098.

20. Kanazawa M, Nakajima S, Oshima T, et al. Validity and Reliability of the Japanese Version of the Rome III Diagnostic Questionnaire for Irritable Bowel Syndrome and Functional Dyspepsia. J Neurogastroenterol Motil. 2015;21:537-544.

21. Kusano M, Hosaka H, Kawada A, et al. Development and evaluation of a modified Frequency Scale for the Symptoms of Gastroesophageal Reflux Disease to distinguish 
functional dyspepsia from non-erosive reflux disease. $J$ Gastroenterol Hepatol. 2012;27:1187-91.

22. Tanaka F, Tominaga K, Fujikawa Y, et al. Association between Functional Dyspepsia and Gastric Depressive Erosions in Japanese Subjects. Intern Med. 2019;58:321-328.

23. Sudo K, Kato K, Matsuzaki J, et al. Development and Validation of an Esophageal Squamous Cell Carcinoma Detection Model by Large-Scale MicroRNA Profiling. JAMA Netw Open. 2019;2:e194573.

24. Asano N, Matsuzaki J, Ichikawa M, et al. A serum microRNA classifier for the diagnosis of sarcomas of various histological subtypes. Nat Commun. 2019;10:1299.

25. Brazma A, Hingamp P, Quackenbush J, et al. Minimum information about a microarray experiment (MIAME)-toward standards for microarray data. Nat Genet. 2001;29:365-371.

26. Kanda Y. Investigation of the freely available easy-to-use software 'EZR' for medical statistics. Bone Marrow Transplant. 2013;48:452-458.

27. Wong N, Wang X. miRDB: an online resource for microRNA target prediction and functional annotations. Nucleic Acids Res. 2015;43:D146-152.

28. Liu W, Wang X. Prediction of functional microRNA targets by integrative modeling of microRNA binding and target expression data. Genome Biol. 2019;20:18.

29. Machaalani R, Chen H. Brain derived neurotrophic factor (BDNF), its tyrosine kinase receptor B (TrkB) and nicotine. Neurotoxicology. 2018;65:186-195.

30. Lu B, Nagappan G, Guan X, Nathan PJ, Wren P. BDNF-based synaptic repair as a diseasemodifying strategy for neurodegenerative diseases. Nat Rev Neurosci. 2013;14:401-416.

31. Tronci E, Napolitano F, Muñoz A, et al. BDNF over-expression induces striatal serotonin fiber sprouting and increases the susceptibility to 1-DOPA-induced dyskinesia in 6-OHDAlesioned rats. Exp Neurol. 2017;297:73-81.

32. Castrén E, Kojima M. Brain-derived neurotrophic factor in mood disorders and 
antidepressant treatments. Neurobiol Dis. 2017;97:119-126.

33. Coulie B, Szarka LA, Camilleri M, et al. Recombinant human neurotrophic factors accelerate colonic transit and relieve constipation in humans. Gastroenterology. 2000;119:41-50.

34. Wang P, Du C, Chen FX, et al. BDNF contributes to IBS-like colonic hypersensitivity via activating the enteroglia-nerve unit. Sci Rep. 2016;6:20320.

35. Yu YB, Zhao DY, Qi QQ, et al. BDNF modulates intestinal barrier integrity through regulating the expression of tight junction proteins. Neurogastroenterol Motil. 2017 Mar;29. doi: $10.1111 /$ nmo.12967. 
Table 1 Clinical characteristics of the study population on microarray analysis

\begin{tabular}{|c|c|c|c|}
\hline Variable & $\mathrm{HC}(\mathrm{n}=6)$ & $\mathrm{FD}(\mathrm{n}=6)$ & $P$-value \\
\hline Age, years & $43.0(41.0,48.8)$ & $43.0(41.0,48.8)$ & 1.000 \\
\hline Male & $3(50.0 \%)$ & $3(50.0 \%)$ & 1.000 \\
\hline BMI, $\mathrm{kg} / \mathrm{m}^{2}$ & $23.2(21.9,23.8)$ & $22.1(19.2,25.0)$ & 0.936 \\
\hline Current cigarette smoking & $1(16.7 \%)$ & $1(16.7 \%)$ & 1.000 \\
\hline Current alcohol drinking & $3(50.0 \%)$ & $2(33.3 \%)$ & 1.000 \\
\hline Frequency of postprandial fullness & $0.0(0.0,0.0)$ & $3.5(3.0,4.8)$ & $<0.01$ \\
\hline Frequency of early satiation & $0.0(0.0,0.0)$ & $0.0(0.0,0.0)$ & 0.405 \\
\hline Frequency of epigastric pain and/or burning & $0.0(0.0,0.0)$ & $4.0(4.0,4.8)$ & $<0.01$ \\
\hline Total score on modified FSSG & $0.5(0.0,1.0)$ & $10.5(9.3,13.3)$ & $<0.01$ \\
\hline Dyspepsia score on modified FSSG & $0.5(0.0,1.0)$ & $7.5(6.3,8.8)$ & $<0.01$ \\
\hline
\end{tabular}

Note: Data are expressed as median (IQR) for continuous variables and as numbers (percentage) for categorical variables.

Abbreviations: BMI, body mass index; FD, functional dyspepsia; FSSG, frequency scale for the symptoms of gastroesophageal

reflux disease; HC, healthy controls; IQR, interquartile range 
Table 2 Results of microarray analysis

\begin{tabular}{|c|c|c|}
\hline Variable & Ratio (FD/HC) & $P_{\text {value }}$ \\
\hline hsa-miR-203a-3p & 0.227 & 0.0187 \\
\hline hsa-miR-3687 & 0.299 & 0.0487 \\
\hline hsa-miR-619-5p & 0.304 & $<0.01$ \\
\hline hsa-miR-3918 & 0.418 & $<0.01$ \\
\hline hsa-miR-4677-5p & 0.430 & 0.0111 \\
\hline hsa-miR-1285-3p & 0.445 & 0.0260 \\
\hline hsa-miR-665 & 0.445 & 0.0280 \\
\hline hsa-miR-1288-3p & 0.446 & 0.0483 \\
\hline hsa-miR-1273a & 0.453 & 0.0246 \\
\hline hsa-miR-4321 & 0.464 & 0.0335 \\
\hline hsa-miR-372-3p & 0.473 & $<0.01$ \\
\hline hsa-miR-4732-5p & 0.516 & 0.0238 \\
\hline hsa-miR-5585-3p & 0.521 & 0.0191 \\
\hline hsa-miR-5096 & 0.524 & 0.0276 \\
\hline hsa-miR-4294 & 0.533 & $<0.01$ \\
\hline hsa-miR-196a-5p & 0.535 & 0.0127 \\
\hline hsa-miR-363-5p & 0.542 & 0.0382 \\
\hline hsa-miR-3667-5p & 0.546 & 0.0333 \\
\hline hsa-miR-3616-3p & 0.563 & 0.0105 \\
\hline hsa-miR-933 & 0.570 & 0.0372 \\
\hline hsa-miR-708-5p & 0.576 & $<0.01$ \\
\hline hsa-miR-3654 & 0.578 & $<0.01$ \\
\hline hsa-miR-3127-5p & 0.583 & 0.0194 \\
\hline
\end{tabular}




\begin{tabular}{lcc} 
hsa-miR-4481 & 0.588 & $<0.01$ \\
hsa-miR-4468 & 0.599 & 0.0264 \\
hsa-miR-3161 & 0.601 & 0.0306 \\
hsa-miR-3649 & 0.604 & 0.0115 \\
hsa-miR-3682-3p & 0.606 & 0.0283 \\
hsa-miR-3158-5p & 0.611 & 0.0268 \\
hsa-miR-4449 & 0.618 & 0.0423 \\
hsa-miR-4800-5p & 0.626 & 0.0171 \\
hsa-miR-6129 & 0.626 & 0.0405 \\
hsa-miR-3135b & 0.628 & 0.0217 \\
hsa-miR-6077 & 0.632 & 0.0385 \\
hsa-miR-7706 & 0.645 & 0.0345 \\
hsa-miR-345-5p & 0.650 & 0.0121 \\
hsa-miR-1273f & 0.650 & 0.0198 \\
hsa-miR-3198 & 0.654 & 0.0172 \\
hsa-miR-658 & 0.659 & 0.0438 \\
hsa-miR-4745-3p & & \\
\hline
\end{tabular}

Note: Ratio represents the signal intensity of FD group divided by that of HC. Abbreviation: HC, healthy controls; FD, functional dyspepsia 
Table 3 Clinical characteristics of the study population by quantitative reverse transcription-PCR

\begin{tabular}{|c|c|c|c|}
\hline Variable & $\mathrm{HC}(\mathrm{n}=5)$ & $\mathrm{FD}(\mathrm{n}=5)$ & $P$-value \\
\hline Age, years & $43.0(35.0,44.0)$ & $43.0(36.0,45.0)$ & 0.917 \\
\hline Male & $3(60.0 \%)$ & $3(60.0 \%)$ & 1.000 \\
\hline BMI, kg/m² & $19.3(18.5,21.9)$ & $21.6(20.6,22.4)$ & 0.690 \\
\hline Current cigarette smoking & $0(0.0 \%)$ & $0(0.0 \%)$ & 1.000 \\
\hline Current alcohol drinking & $4(80.0 \%)$ & $3(60.0 \%)$ & 1.000 \\
\hline Frequency of postprandial fullness & $0.0(0.0,1.0)$ & $4.0(4.0,5.0)$ & $<0.01$ \\
\hline Frequency of early satiation & $0.0(0.0,0.0)$ & $0.0(0.0,5.0)$ & 0.177 \\
\hline Frequency of epigastric pain and/or burning & $0.0(0.0,0.0)$ & $4.0(4.0,5.0)$ & $<0.01$ \\
\hline Total score on modified FSSG & $2.0(0.0,3.0)$ & $17.0(17.0,23.0)$ & 0.0117 \\
\hline Dyspepsia score on modified FSSG & $1.0(0.0,1.0)$ & $11.0(8.0,13.0)$ & 0.0117 \\
\hline
\end{tabular}

Note: Data are expressed as median (IQR) for continuous variables and as numbers (percentage) for categorical variables.

Abbreviation: BMI, body mass index; FD, functional dyspepsia; FSSG, frequency scale for the symptoms of gastroesophageal

reflux disease; HC, healthy controls; IQR, interquartile range 
Table 4 Validation of microarray analysis results by quantitative reverse transcription-PCR

\begin{tabular}{llll}
\hline Variable & HC $(\mathrm{n}=5)$ & FD $(\mathrm{n}=5)$ & $P$ value \\
\hline hsa-miR-933 & $1.000(0.522)$ & $0.317(0.205)$ & 0.0317 \\
hsa-miR-345-5p & $1.000(1.173)$ & $0.338(0.387)$ & 0.421 \\
hsa-miR-708-5p & $1.000(0.970)$ & $0.383(0.493)$ & 0.151 \\
hsa-miR-203a-3p & $1.000(0.627)$ & $0.478(0.229)$ & 0.151 \\
hsa-miR-619-5p & $1.000(0.303)$ & $0.604(0.295)$ & 0.0556 \\
hsa-miR-4294 & $1.000(0.252)$ & $0.709(0.605)$ & 0.548 \\
hsa-miR-4745-3p & $1.000(0.451)$ & $0.734(0.589)$ & 0.421 \\
hsa-miR-4481 & $1.000(0.375)$ & $0.974(0.239)$ & 0.841 \\
hsa-miR-196a-5p & $1.000(1.903)$ & $0.975(1.686)$ & 0.841 \\
hsa-miR-3918 & $1.000(0.516)$ & $1.076(0.287)$ & 0.690 \\
hsa-miR-372-3p & $1.000(0.567)$ & $1.447(0.678)$ & 0.421 \\
hsa-miR-658 & $1.000(0.461)$ & $1.878(0.664)$ & 0.0952 \\
hsa-miR-3654 & $1.000(0.536)$ & $2.160(1.893)$ & 0.421 \\
\hline Note: & &
\end{tabular}

Note: Data are expressed as mean (standard deviation).

Abbreviation: FD, functional dyspepsia; HC, healthy controls 
Fig. 1

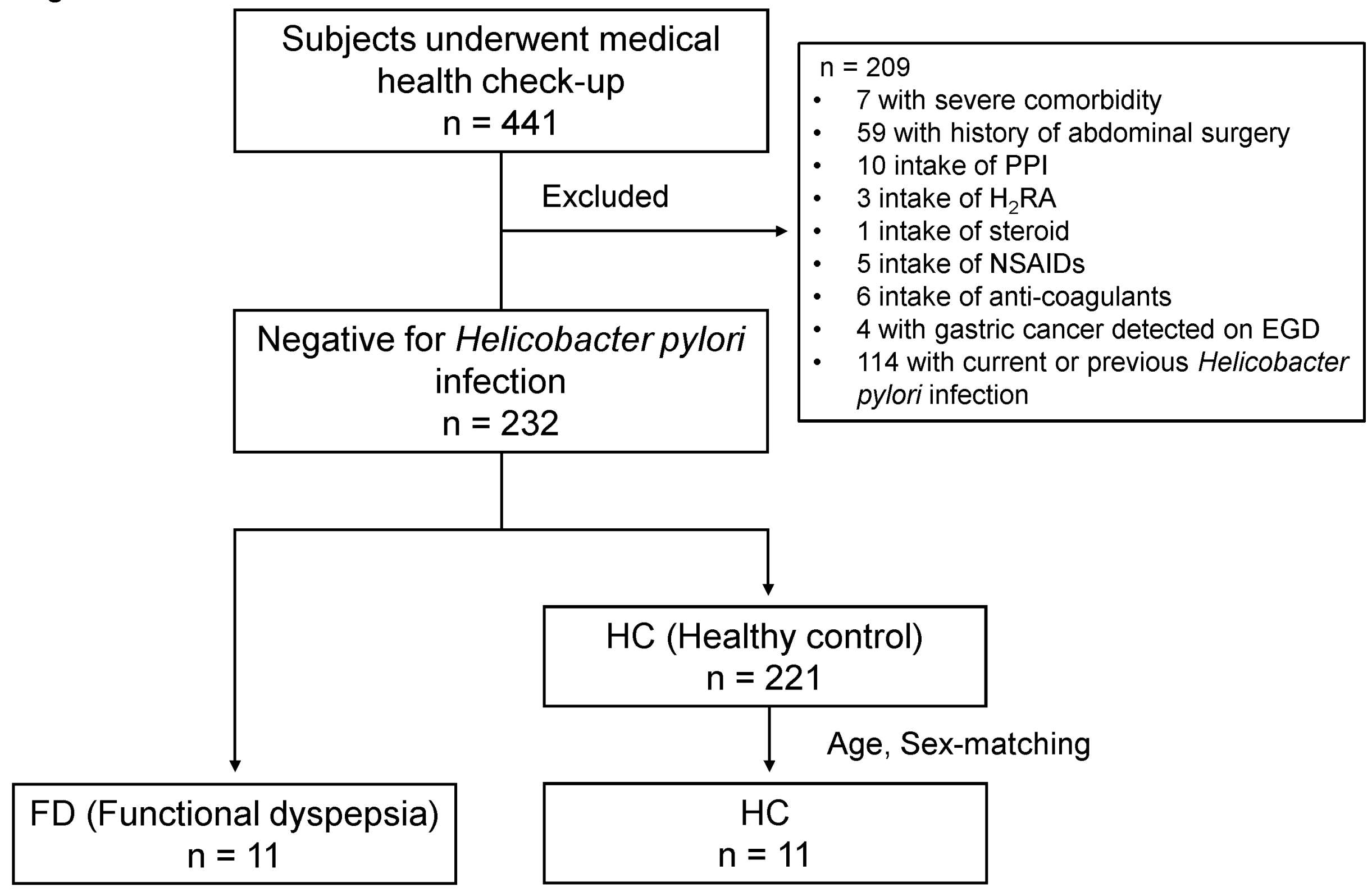


Fig. 2
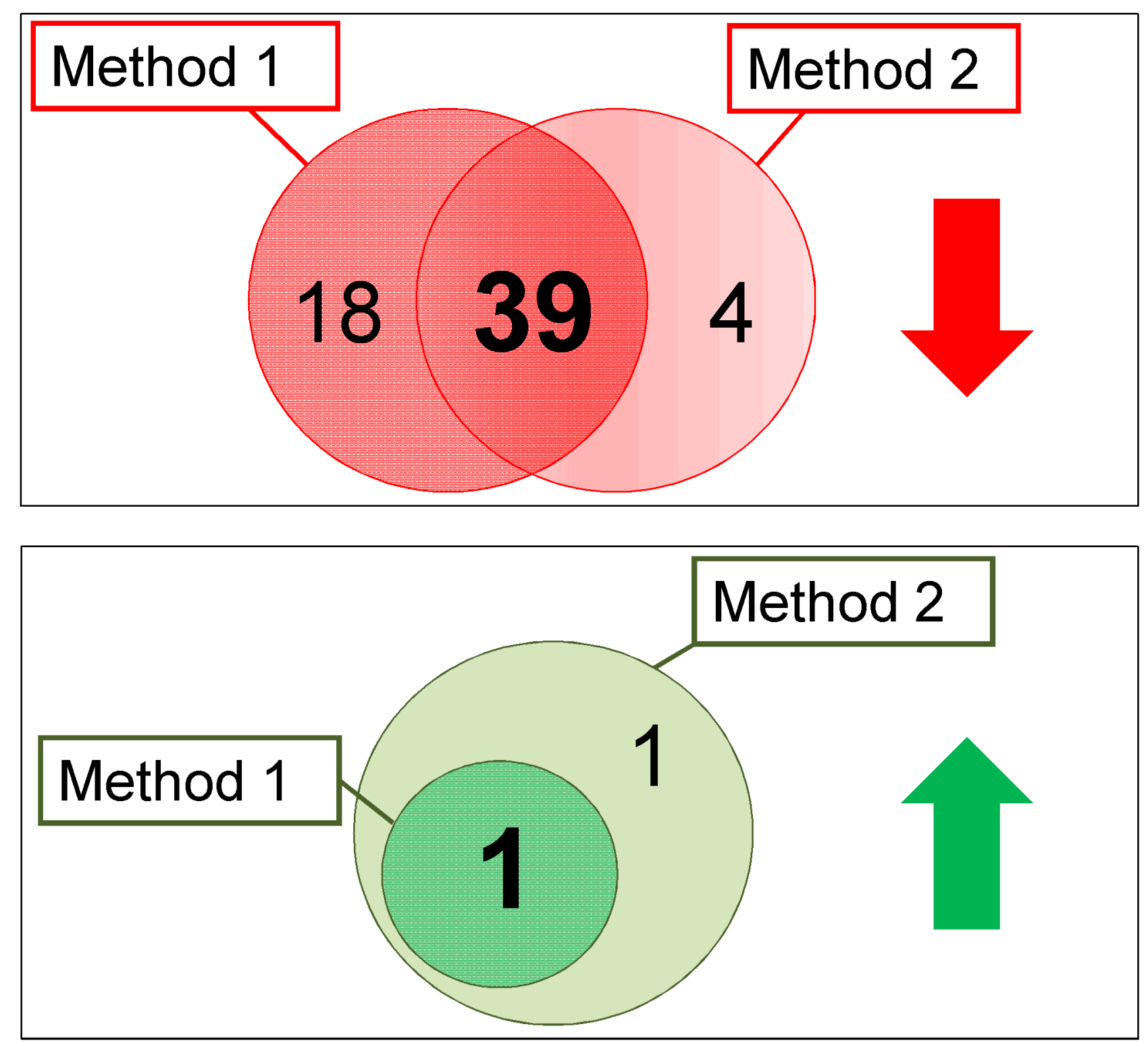
Fig. 3

A

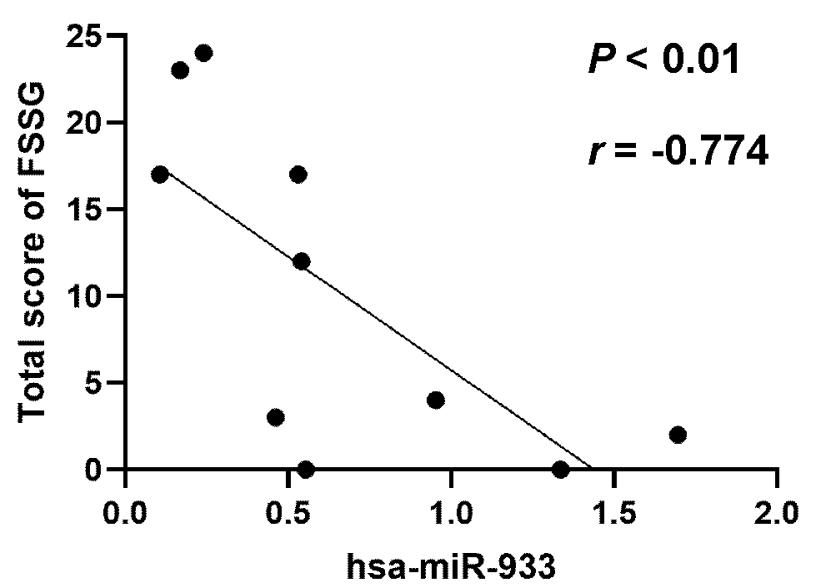

C

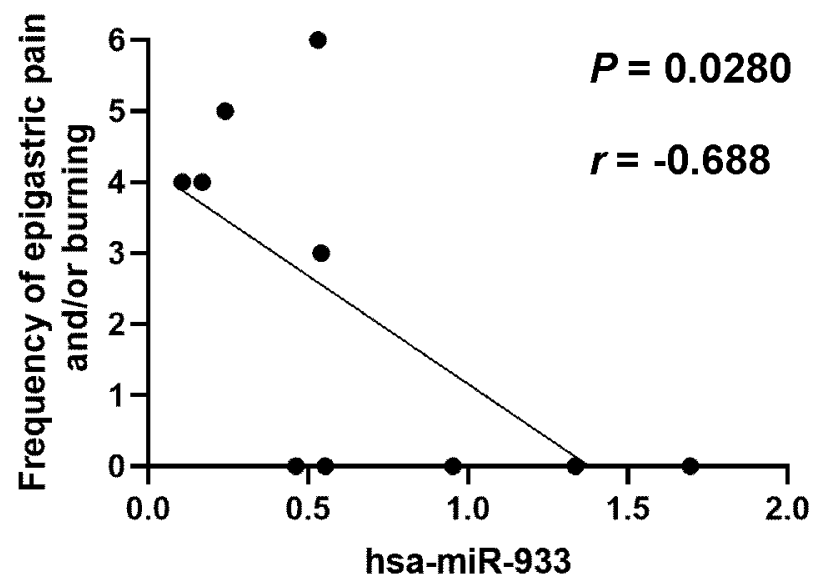

B

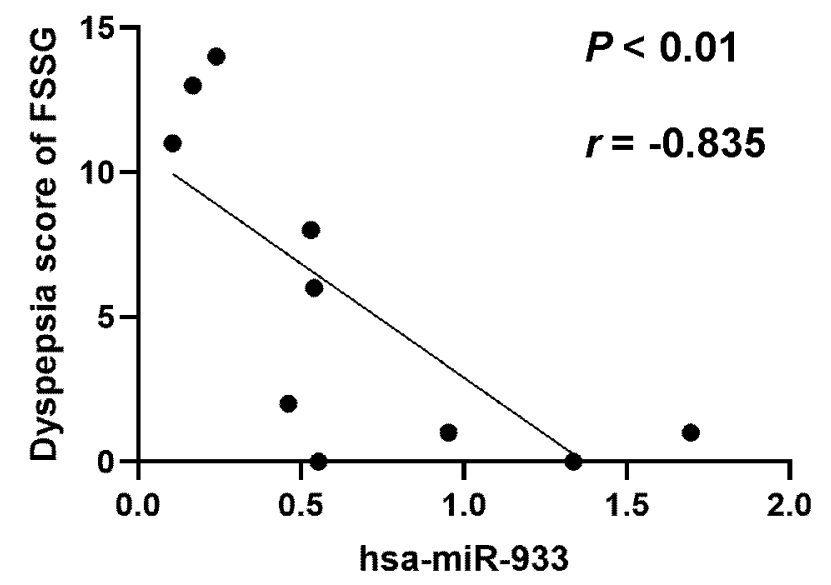

D

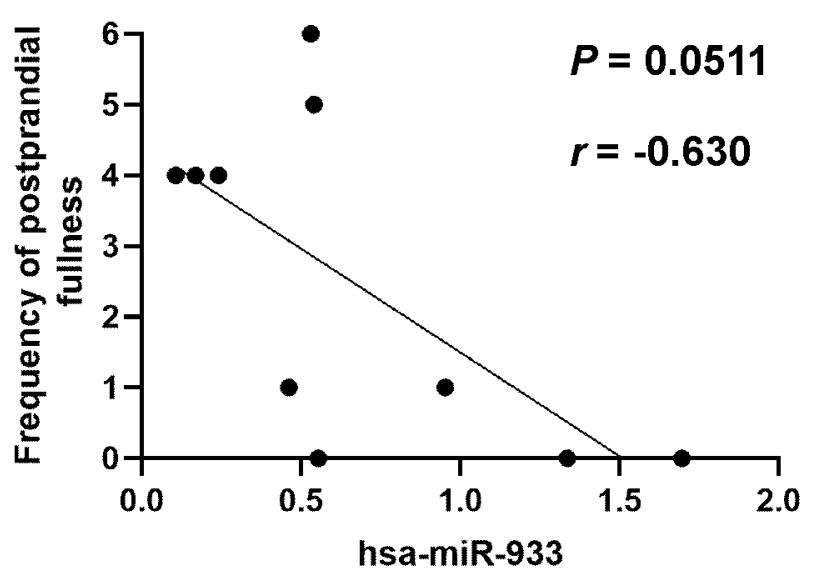

